\title{
Scattering of electrically excited surface plasmon polaritons by gold nanoparticles studied by optical interferometry with a scanning tunneling microscope
}

\author{
Tao Wang,,${ }^{1, *}$ Benoît Rogez, ${ }^{1}$ Geneviève Comtet,${ }^{1}$ Eric Le Moal,${ }^{1, \dagger}$ Wafa Abidi, ${ }^{2}$ Hynd Remita, ${ }^{2}$ \\ Gérald Dujardin, ${ }^{1}$ and Elizabeth Boer-Duchemin ${ }^{1}$ \\ ${ }^{1}$ Institut des Sciences Moléculaires d'Orsay, CNRS-Université Paris-Sud (UMR 8214), Orsay, France \\ ${ }^{2}$ Laboratoire de Chimie Physique, CNRS-Université Paris-Sud (UMR 8000), Orsay, France \\ (Received 9 March 2015; revised manuscript received 17 April 2015; published 31 July 2015)
}

\begin{abstract}
We study the scattering of electrically excited surface plasmon polaritons (SPP) from individual nanostructures. The tunneling electrons from a scanning tunneling microscope (STM) are used to excite an out-going, circular SPP wave on a thin (50-nm) gold film on which isolated gold nanoparticles (NPs) have been deposited. Interaction of the excited SPPs with the NPs leads to both in-plane (SPP-to-SPP) and out-of-plane (SPP-to-photon) scattering. We use SPP leakage radiation microscopy to monitor the interference between the incident and in-plane scattered SPP waves in the image plane. By changing the location of the STM tip, the distance of the pointlike SPP source to the scatterers can be varied at will, which constitutes a key advantage over other existing techniques. As well, the out-of-plane scattered radiation interferes with the direct light emission from the STM tip in the back focal plane (Fourier plane). This confirms the mutual coherence of the light and SPP emission resulting from the inelastic tunneling of an electron in the STM junction. We use this effect to demonstrate that SPP-to-photon scattering at NPs is highly directional.
\end{abstract}

DOI: 10.1103/PhysRevB.92.045438

PACS number(s): 73.20.Mf, 68.37.Ef, 78.67.Bf

\section{INTRODUCTION}

Surface plasmon polaritons (SPPs), i.e., electromagnetic waves propagating on a metallic surface, are considered key elements for the integration of optics with nanoelectronics [1-5]. In plasmonic circuitry, SPP propagation can be controlled via in-plane SPP-to-SPP scattering on a nanostructured metallic surface [6-8]. Efficient scatterers such as metal nanoparticles (NPs) may be assembled in periodic arrays to form microoptical components for SPPs, e.g., mirrors, beam splitters, and lenses [9-13]. Importantly for optoelectronics applications, out-of-plane SPP-to-photon scattering at NPs may be used to engineer the coupling between SPPs and light [14,15].

Several experimental studies on the NP-scattering of SPPs have been reported [16-25], most of them based on scanning near field optical microscopy (SNOM) [16-23]. Crucial aspects such as coherence conservation and phase shifts upon SPP scattering by metal NPs have, however, rarely been probed so far. As well, the angular distribution of out-of-plane SPP scattering is an important observable that is often not accessible in the reported experimental approaches. Cathodoluminescence (CL) imaging techniques have been developed to angularly resolve the light emission from single gold nanodisks [26] or nanoholes [27] upon direct excitation of their plasmonic modes with a high-energy electron beam; yet, this technique has not been applied to the study of the scattering of propagating SPPs by a single nanostructure. A high-energy electron beam has also been used to excite propagating SPPs directly on unstructured metal surfaces and out-coupled into photons using a grating, thus leading to a measurement of the plasmon propagation length [28,29]. As well, phase-sensitive SNOM (P-SNOM) techniques have been

\footnotetext{
*Present address: Department of Chemistry, NUS 3 Science Drive 3, Singapore 117543.

†Corresponding author: eric.le-moal@u-psud.fr
}

used to characterize the response of a hole etched in a thick gold film to incident SPP plane waves [30,31]. Complex-amplitude nearfield images are measured by raster scanning a nearfield detection probe and the angular distribution of both the SPP and light fields can in principle be retrieved by computing the Fourier transform of these images; however, the influence of the nearfield probe has been shown to introduce artifacts [30]. So far, no P-SNOM study of SPP scattering by a NP has been reported. A widefield imaging mode where the whole region of interest is imaged simultaneously is ideal for the study of NP scattering of SPPs. In conjunction with local SPP excitation, widefield imaging may be used to monitor the spatial distribution of the extended SPP modes as well as their interplay with local scatterers $[11,18]$. Photoemission electron microscopy (PEEM) is also a technique that has widefield imaging capabilities. An interferometric time-resolved technique derived from PEEM has recently been used to study the interaction of SPPs with gold nanodots [25]. In this technique, the SPP waves are optically generated by coupling light from the far field to a fixed step edge on the gold surface. In this configuration, SPP scattering at a given nanostructure can only be characterized for a single direction of SPP incidence and a single SPP source-nanodot distance (virtually infinity since SPP plane waves are generated). Moreover, in-plane and out-of-plane SPP scattering cannot be distinguished with PEEM.

In this article, we combine optical and scanning tunneling microscopy (STM) to study the SPP scattering properties of individual nanostructures. This technique combines the advantages of a local SPP source (like SNOM and CL) and widefield optical imaging. Such an experimental approach has recently been used to study SPP scattering at the end of a gold nanowire [32], at the edges of a gold nanostripe [33], at holes etched in a gold film [34,35], and at the slits of linear [36] and circular gratings [37]; yet this approach has never been used to study the interaction of 2D SPPs with a single OD NP. In this experiment, we use the inelastic tunneling of electrons from 

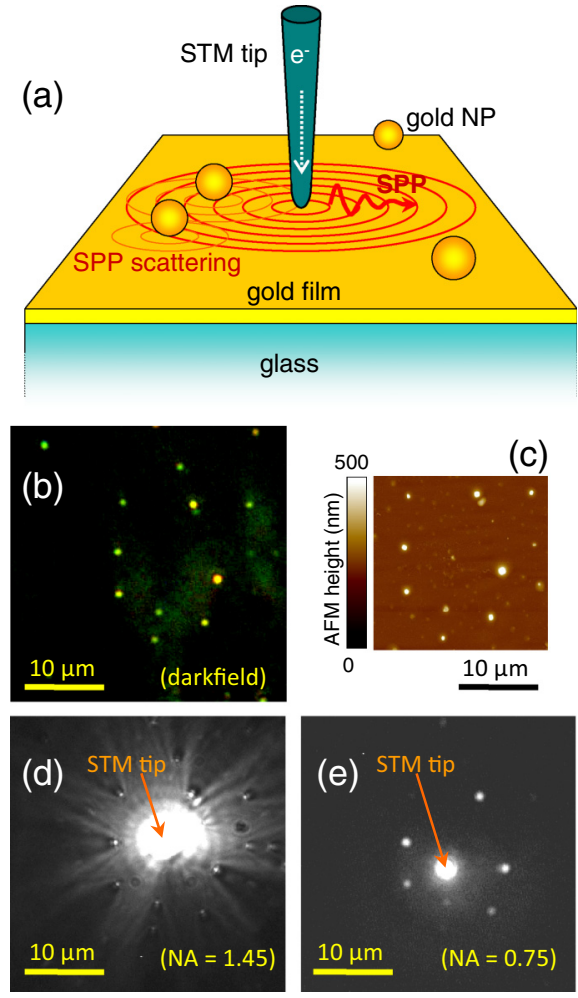

FIG. 1. (Color online) (a) Schematic of the experiment: gold nanoparticles (NPs) are dispersed on a thin gold film deposited on a glass coverslip. Inelastic electron tunneling from the STM tip to the gold film electrically excites surface plasmon polaritons (SPPs) that propagate isotropically away from the tunnel junction. SPP-to-SPP scattering at the gold NPs is imaged by leakage radiation microscopy (LRM), using an inverted optical microscope coupled to the STM. (b) Darkfield optical microscopy image of gold NPs dispersed on the gold film. This image is obtained using an air objective of numerical aperture NA $=0.75$ upon illumination of the sample with a collimated white light beam at grazing incidence. (c) Atomic force microscopy (AFM) image of the same area as in (b). (d) Gray-scale optical LRM image of the same area during STM-SPP excitation, obtained using an oil-immersion objective of NA $=1.45$. The location of the STM tip on the gold film is indicated by the red arrow. (e) Same as in (d) but obtained using an air objective of NA $=0.75$. Such an objective cannot collect the SPP leakage radiation.

the STM tip as a nanosource of circular SPP waves on a thin gold film [32,38]. These waves propagate away from the tip location and scatter at gold NPs on the gold film [see Fig. 1(a)]. Two different elastic-scattering processes may occur: in-plane SPP-to-SPP scattering (NPs act as secondary sources of SPPs) and out-of-plane SPP-to-photon scattering [21]. Both processes are investigated by monitoring the light transmitted through the substrate, which is collected using a microscope objective and imaged on a cooled charge-coupled device camera. The interference between the incident and in-plane scattered SPPs is recorded by imaging the leakage radiation in real space (spatial distribution) [18,39]. In Fourier space (angular distribution), we examine the optical interference between the scattering radiation from the NPs and the light directly emitted from the STM tip. We find that SPPs scattered out-of-plane by gold NPs are highly forward scattered. Finally, our results demonstrate that all the emission and scattering processes at play, namely the light and SPP emission from the STM tip and the in-plane and out-of-plane scattering at the NPs, are mutually coherent.

\section{METHODS}

We use an STM-AFM (atomic force microscope) head (Veeco Bioscope/Nanoscope IVa) mounted onto an inverted optical microscope (Zeiss Axiovert). A nanopositioning stage controls the lateral position of the sample with respect to the STM tip, which is an electrochemically etched tungsten wire. Gold NPs (mean diameter $150 \mathrm{~nm}$ ) stabilized by cyanide ligands are synthesized using a seed-mediated method by radiolysis [40] and drop casted onto a thin gold film (thickness $50 \mathrm{~nm}$ ) thermally evaporated on a glass coverslip. The sample is biased to $2.8 \mathrm{~V}$ with respect to the tip and the setpoint tunnel current is $6 \mathrm{nA}$.

\section{RESULTS AND DISCUSSION}

Figure 1 illustrates the principle of our approach and highlights its advantages for the study of SPP scattering. Prior to STM experiments, we detect and identify the same gold NPs on the gold film by both darkfield optical microscopy [see Fig. 1(b)] and tapping-mode AFM [see Fig. 1(c)]. Subsequently, we use the STM tip to generate a pointlike source [34] of 2D circular SPPs in the same region of interest. Figure 1(d) shows the real-space image obtained with an oil-immersion objective (NA $=1.45, \theta_{\max }=72^{\circ}$ in glass). With such an objective, the SPP leakage radiation from the air-gold interface, which is emitted at an angle that is slightly larger than the air-glass critical angle $\left(\theta_{c}=41^{\circ}\right)$, is collected. The coherent superposition of the incident and scattered SPP waves generates a hyperbolic dark "shadow" just after the gold NPs [see Fig. 1(d)]. Figure 1(e) shows the real-space image measured under the same conditions but with an air objective $\left(\mathrm{NA}=0.75, \theta_{\max }=30^{\circ}\right.$ in glass), whose numerical aperture is too low to collect the SPP leakage radiation. Bright spots are seen at the location of the gold NPs since the light from SPP out-of-plane scattering is emitted at all angles. The presence of an intense spot at the tip location is not due to secondary scattering of back-scattered SPP waves at the tip but results from the fact that the tunnel junction behaves as both a nanosource of SPPs and photons. This is expected since STM-induced light emission is often described as the radiation of a vertical electric dipole inside the tunnel junction [41]. Close to a thin gold film on glass, a vertical electric dipole couples naturally to both SPPs bound to the air-gold interface as well as to free-space radiation in air and glass.

In the following we report on experiments involving a single, isolated NP. Figure 2 shows that a hyperbolic dark "shadow" forms in the real-space image when SPPs elastically scatter into SPPs at a gold NP. This feature is particularly interesting because it is related to the phase shift between the incident and scattered SPP waves. As well, the tip-NP distance $d$ has a strong effect on the in-plane scattering angle $2 \Psi$, i.e., the angular aperture of the "shadow" after the NP. We measure several real-space images of the same 

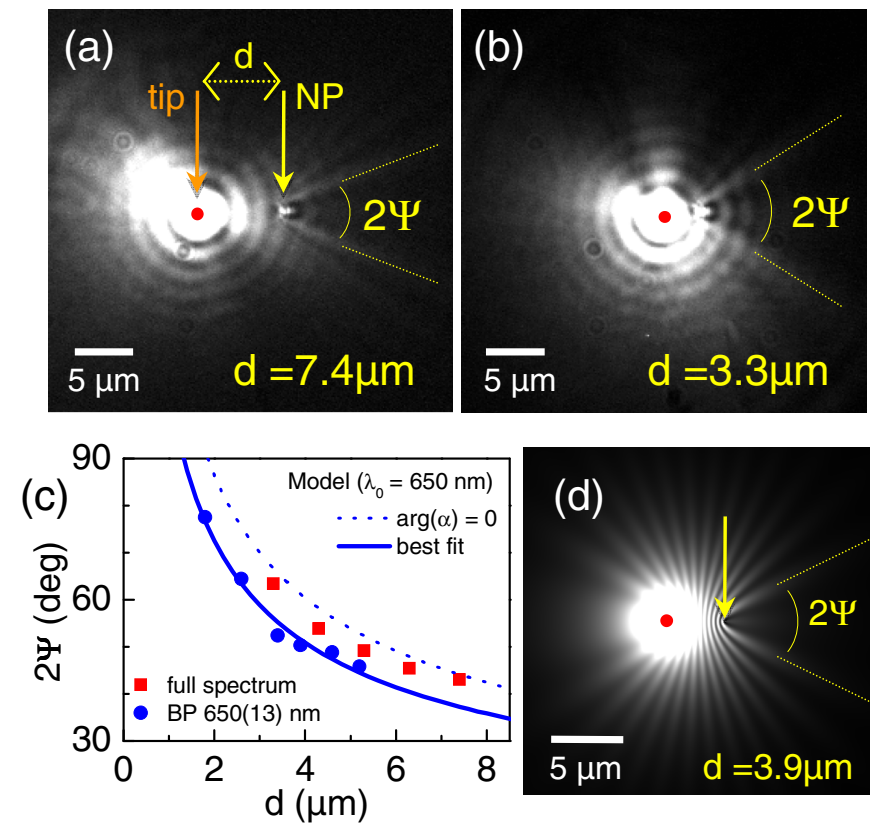

FIG. 2. (Color online) In-plane SPP-to-SPP scattering by a single NP studied in real space. [(a) and (b)] Gray-scale optical LRM images of SPP scattering after STM-SPP excitation on a gold film for two different tip-NP separation distances $d$ (acquired using an oil-immersion objective of NA $=1.45$ ). The location of the tip and $\mathrm{NP}$ are indicated by a red dot and a yellow arrow, respectively. The scattering angle $2 \Psi$ defines the angular aperture of the hyperbolic dark "shadow" that is seen after the NP. Image acquisition time is $120 \mathrm{~s}$ and the full broadband emission spectrum is used. The rings seen in these experimental images are due to the point spread function of the diffraction-limited microscope optics [42]. (c) Experimental and theoretical dependence of the scattering angle $2 \Psi$ on the tip-NP separation distance $d$. (d) Theoretical (nearfield) distribution of the SPP electric field intensity on the gold film for $\lambda_{0}=650 \mathrm{~nm}$, $d=3.9 \mu \mathrm{m}$, and $\arg (\alpha)=\frac{\pi}{2}$. Theoretical data in (c) and (d) are calculated within the simple scalar model of a pointlike isotropic scatterer of effective polarizability $\alpha$ [21].

area with tip-NP separations ranging from $d=1.8 \mu \mathrm{m}$ to $d=$ $7.4 \mu \mathrm{m}$, either using the full broadband emission spectrum (centered on wavelength $700 \mathrm{~nm}$ and about $200 \mathrm{~nm}$ wide at half maximum [38]) as in Figs. 2(a) and 2(b) or a filtered narrow spectral band around $650 \mathrm{~nm}(13 \mathrm{~nm}$ wide, image not shown). From the analysis of such images, we retrieve in-plane scattering angles ranging from $2 \Psi=77.5^{\circ}$ to $2 \Psi=43^{\circ}$, as shown in Fig. 2(c). We find that the closer the tip is to the NP, the larger the angle $\Psi$. As well, we see that $\Psi$ is smaller when the narrow-pass filter at $650 \mathrm{~nm}$ is used. The dependence of $\Psi$ on $d$ may be understood qualitatively by considering that changing the tip location modifies the curvature of the SPP wavefront impinging on the NP, which inevitably changes the interference pattern arising from the superposition of the incident and scattered SPP waves.

Bozhevolnyi and Coello [21] have proposed a scalar model for the in-plane scattering of SPP plane waves at an NP, where the NP is considered a pointlike isotropic scatterer of effective polarizability $\alpha$. As discussed by Evlyukhin and Bozhevolnyi $[43,44]$, the point-dipole and scalar approxima- tions are valid only for very small NPs as compared to the SPP wavelength, especially when considering gold NPs in close proximity to a gold substrate. Nevertheless, this simple model is useful for the understanding of the general features of the interference pattern formed between the incident and scattered SPP waves. We adapt this model to 2D circular SPP waves (see Appendix A) and use it to explain the dependence of the in-plane SPP scattering angle on the tip-NP distance.

Within this model, the SPP field intensity $I(\mathbf{r})$ at an arbitrary surface point $\mathbf{r}$ may be written as a sum of three terms corresponding to the SPP fields emitted from the tip, from the NP and their interference, respectively:

$$
I(\mathbf{r})=I_{\text {tip }}\left(d_{1}\right)+I_{\mathrm{NP}}\left(d_{2}\right)+I_{\text {int }}\left(d_{1}, d_{2}\right),
$$

where $d_{i}=\left|\mathbf{r}-\mathbf{r}_{\mathbf{i}}\right|$ and $\mathbf{r}_{1}$ and $\mathbf{r}_{2}$ are the tip and NP locations, respectively. As an example, Fig. 2(d) shows the theoretical distribution of $I(\mathbf{r})$ (i.e., the modulus squared of the electric field on the surface of the film) for $d=3.9 \mu \mathrm{m}, \lambda_{0}=650 \mathrm{~nm}$, and $\arg \alpha=\frac{\pi}{2}$. From the expression of $I_{\text {int }}$ (see Appendix A), one finds that bright fringes exist in the interference pattern whenever $d_{1}-$ $d_{2}=d+\frac{1}{k^{\prime}}\left[2 \pi p+\frac{\pi}{4}+\arg (\alpha)-\frac{1}{2} \arg \left(k_{\mathrm{SPP}}\right)\right]$, where $k_{\mathrm{SPP}}=$ $k^{\prime}+i k^{\prime \prime}$ is the complex SPP wave number and $p$ is an integer. As a result, the interference of the incident and scattered SPP waves yields a pattern of hyperbolic fringes. The angle $2 \Psi$ between the asymptotes of the bright fringes is given by $\cos (\Psi)=\frac{d_{1}-d_{2}}{d}$, i.e., $\cos (\Psi)=$ $1+\frac{1}{k^{\prime} d}\left[2 \pi p+\frac{\pi}{4}+\arg (\alpha)-\frac{1}{2} \arg \left(k_{\mathrm{SPP}}\right)\right]$. This elucidates the dependence of the in-plane scattering angle on the tip-NP separation, $d$. As seen in Fig. 2(c), we find good agreement between the theoretical and experimental values of $2 \Psi$ by adjusting the single fitting parameter $\arg (\alpha)$ to $1.55 \mathrm{rad}$. If the point-dipole and scalar approximations were rigorously valid, $\arg (\alpha)$ would directly provide the phase relation between the charge oscillations in the NP and the incident SPP wave. In any case, the measured interference patterns shown in Figs. 2(a) and 2(b) contain the necessary information to retrieve the scattering matrix which relates the complex amplitude of the incident and scattered SPP waves far from the NP [45]; yet, a full vectorial multipolar treatment is required to obtain the polarizability dyadic $\stackrel{\leftrightarrow}{\alpha}$ of the NP [46].

We now focus on the back-focal-plane measurements, where can be seen the interference between out-of-plane SPP scattering and direct light emission from the STM tip. Figure 3 shows Fourier images obtained for tip-NP separation distances of $d=5.2 \mu \mathrm{m}$ and $3.6 \mu \mathrm{m}$ (objective NA $=1.45$, bandpass filter at $\lambda_{0}=650 \mathrm{~nm}$ ). A narrow, intense light ring is observed close to the critical angle. This corresponds to SPP leakage radiation. In addition, the Fourier images exhibit linear interference fringes that are perpendicular to the tip-NP axis. The fringes are seen above the critical angle and up to the maximum acceptance angle of the objective lens. As well, they only occur in the tip-to-NP direction, within an azimuthal range estimated at about $90^{\circ}$, whatever $d$. This demonstrates that out-of-plane SPP scattering by a 150-nm gold NP is highly directional (no detectable backward out-of-plane scattering observed).

In Fig. 3(e), we see that the fringe period $\Delta k_{x}$ in Fourier space increases when the tip-NP distance is decreased. $\Delta k_{x}$ 

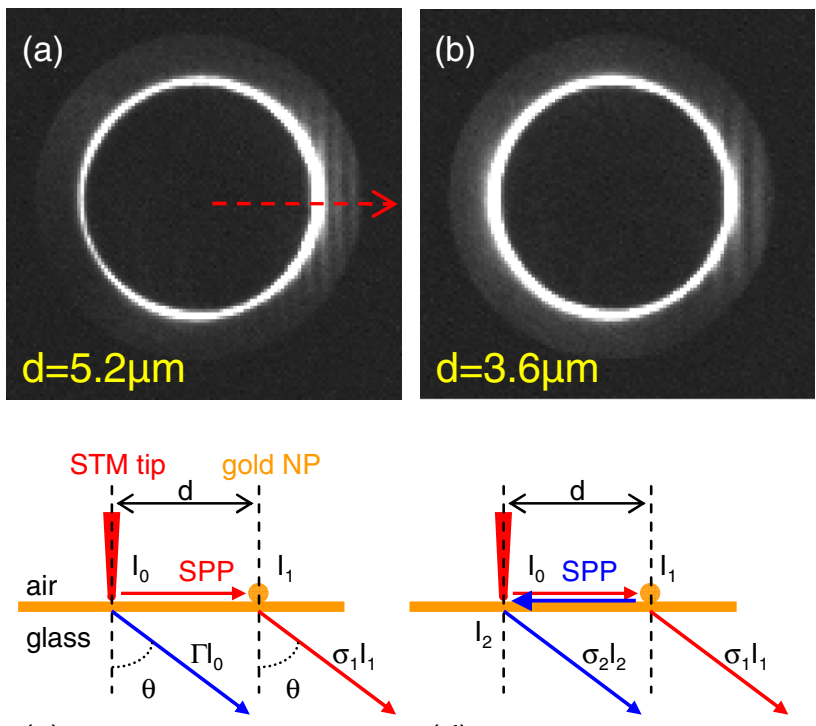

(c)
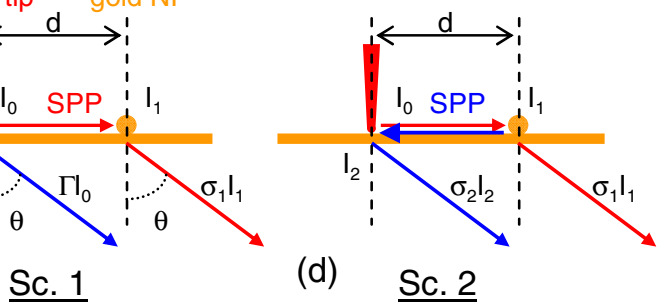

(d)
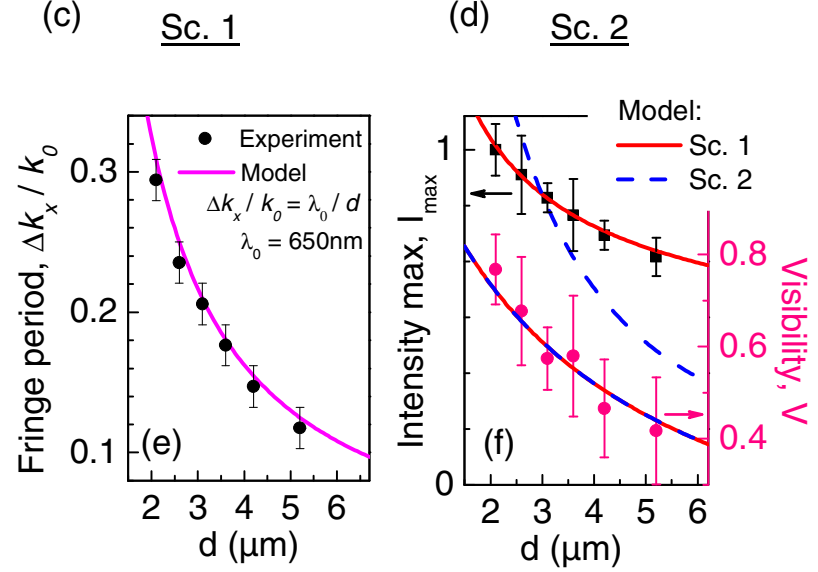

FIG. 3. (Color online) Out-of-plane SPP-to-photon scattering by a single NP studied in Fourier space. [(a) and (b)] Gray-scale optical LRM images measured in Fourier space for two different tip-NP distances (exposure time: $120 \mathrm{~s}$, bandpass filter at $\lambda_{0}=$ $650 \mathrm{~nm}$ ). The red dashed arrow indicates the tip-to-NP direction. [(c) and (d)] Schematics of two possible scenarios that may explain the interference fringes observed in Fourier space (see the text for parameter definitions). [(e) and (f)] Experimental and theoretical dependence of (e) the fringe period, (f) maximal intensity (black squares), and visibility (magenta dots) on the tip-NP separation $d$. The distance dependence of $I_{\max }$ validates scenario 1 and rules out scenario 2 .

closely follows a $\Delta k_{x} / k_{0}=\lambda_{0} / d$ rule, which is typical of the interference of light from two pointlike coherent sources [34]. The two coherent light sources are the tunnel junction and the NP; yet two possible scenarios (or propagation paths) may explain our observations, as illustrated in Figs. 3(c) and 3(d). In scenario 1 (Sc. 1), out-of-plane forward SPP scattering at the NP interferes with the directly emitted light from the tunnel junction. In scenario 2 (Sc. 2) the out-of-plane forward SPP scattering at the NP interferes with the SPP wave that has been scattered twice: first at the NP (in-plane backward scattering) and then at the tip (out-of-plane backward scattering). Importantly, Sc. 1 requires that the plasmonic and photonic emission of the tunnel junction be mutually coherent, whereas Sc. 2 does not, since it involves the same SPP that is elastically and coherently scattered in the plane. We propose below a simple method to determine the correct scenario, which takes advantage of the fact that we can easily vary the tip-NP distance.

We define $I_{0}$ as the initial intensity of the electric field at the STM-tip nanosource, $I_{1}$ as the intensity at the NP after SPP propagation from the tip, and $I_{2}$ as the intensity at the tip after SPP propagation back from the NP. In Fourier space, the intensity of the interference fringes is $I\left(k_{x}\right)=\Gamma I_{0}+$ $\sigma_{1} I_{1}+2 \sqrt{\Gamma I_{0} \sigma_{1} I_{1}} \cos \left(k_{x} d+\Delta \phi_{1}\right)$ in Sc. 1 and $I\left(k_{x}\right)=$ $\sigma_{1} I_{1}+\sigma_{2} I_{2}+2 \sqrt{\sigma_{1} I_{1} \sigma_{2} I_{2}} \cos \left(k_{x} d+\Delta \phi_{2}\right)$ in Sc. 2, where $\Gamma$ is the relative efficiency of the light emitted directly by the STM-nanosource in the substrate, $\sigma_{i}$ is the out-of-plane SPP scattering efficiency at the NP $(i=1)$ or at the tip $(i=2)$, and $\Delta \phi_{i}$ is the phase shift due to SPP propagation [35] and out-of-plane scattering. As a result, the intensity of the bright and dark fringes is $I_{\max } / \min =\Gamma I_{0}+\sigma_{1} I_{1} \pm 2 \sqrt{\Gamma I_{0} \sigma_{1} I_{1}}$ in Sc. 1 and $I_{\max / \min }=\sigma_{1} I_{1}+\sigma_{2} I_{2} \pm 2 \sqrt{\sigma_{1} I_{1} \sigma_{2} I_{2}}$ in Sc. 2 , respectively. We note $V=\frac{I_{\max }-I_{\min }}{I_{\max }+I_{\min }}$ the visibility of the fringe pattern. In Fig. 3(f), we plot the experimental values of $I_{\max }$ and $V$ versus $d$ and fit them with their theoretical expressions (fitting parameters are $I_{0}, \Gamma$, and $\sigma_{i}$ ). $I_{1}$ and $I_{2}$ are calculated by assuming emission of cylindrical, scalar SPP waves from the tip and NP locations (see Appendix B). It is clear in Fig. 3(f) that very good agreement between experiment and theory is similarly obtained for $V$ in both scenarios. However, $I_{\max }$ varies quite differently with the tip-NP distance $d$; indeed, $I_{\max }=\Gamma I_{0}\left(1+\sqrt{\frac{\eta_{1}}{d}} e^{-k^{\prime \prime} d}\right)^{2}$ in Sc. 1 , whereas in Sc. $2 I_{\max }=\sigma_{1} I_{0} \frac{e^{-2 k^{\prime \prime} d}}{d}\left(1+\sqrt{\frac{\eta_{2}}{d}} e^{-k^{\prime \prime} d}\right)^{2}$ where $\eta_{i}(i=1,2)$ is a constant (see Appendix B). As seen in Fig. 3(f), $I_{\max }$ closely reproduces the experimental observations in Sc. 1, unlike in Sc. 2 (whose least-squares fit leads to value of $\sigma_{2}=0$ ). Therefore Sc. 2 may be ruled out and the scenario where the SPPs scattered at the NP interfere with the light directly emitted by the tunnel junction is validated. These results are conclusive evidence that the plasmonic and photonic emission channels of the tunnel junction are coherent. Our observations also verify that this coherence is preserved when the propagating SPPs are converted into photons upon elastic scattering at a nanostructure, otherwise no interference would occur in the Fourier plane.

\section{CONCLUSION}

To conclude, we combine STM with optical microscopy to study the SPP scattering properties of gold NPs on a gold film. Electrically excited SPPs are launched from the tunnel junction of an STM, yielding an isotropic nanosource of circular SPP waves. This approach provides both the advantages of a local, electrical SPP excitation with a widefield optical imaging mode. In this way, the spatial distribution of the extended SPP modes and their interaction with localized scatterers may be monitored. The SPP STM-nanosource can be moved to any point on the gold surface that is accessible by STM, unlike other techniques which use a fixed defect or grating to optically excite SPPs. We study how the distance $d$ between the SPP nanosource and a single NP affects the interference pattern 
that forms in real and Fourier-space images. In real space, hyperbolic fringes arise from the coherent superposition of the incident and in-plane scattered SPP waves which are detected via SPP leakage radiation. In Fourier space, linear fringes are observed at supercritical angles in the tip-to-NP direction, due to the directionality of the out-of-plane scattering at the NP. The fringe period dependence on $d$ demonstrates that the SPPs radiatively scattered at the NP interfere with the light directly emitted from the tunnel junction. Thus, we experimentally demonstrate the mutual coherence of the photonic and plasmonic emission from the inelastic tunneling of an electron in the STM junction.

The mutual coherence of the SPP and light emission resulting from the inelastic tunneling of an electron through the tunnel junction is a direct consequence of the wave nature of SPPs and photons. Electron tunneling is indeed a stochastic process and no coherence is expected between two successive tunneling events. From a classical point of view, we may thus consider that one single inelastic electron tunneling event yields an electromagnetic wave that simultaneously propagates along the air-gold interface and in the glass substrate, i.e., a wave that has both plasmonic and photonic character.

These STM-based interferometric measurements may be used in future work to monitor SPP coherence losses and phase shifts in more complicated systems, including semicontinuous metallic films $[47,48]$ and hybrid systems where SPPs are strongly coupled to the excitons of semiconductor nanostructures $[49,50]$.

\section{ACKNOWLEDGMENTS}

This work was supported by Agence Nationale de la Recherche through project NAPHO (Contract No. ANR-08NANO-054) and by the European Commission through the Seventh Framework Programme (FP7-ICT-2009, Contract No. 243421). We thank C. Girard, J.-J. Greffet, and J.-P. Hugonin for fruitful discussions.

\section{APPENDIX A: MODEL FOR IN-PLANE (SPP-TO-SPP) SCATTERING}

In order to understand the general features of the interference pattern of the incident and scattered SPP waves, we use a scalar model first proposed by Bozhevolnyi and Coello for the scattering of SPP plane waves [21]. Here we adapt this model to $2 \mathrm{D}$ circular SPP waves. The NP is considered a pointlike isotropic scatterer of effective polarizability $\alpha$. The scalar SPP field $E(\mathbf{r})$ at an arbitrary surface point $\mathbf{r}$ is the coherent sum of the isotropic SPP emission from the tunnel junction and the scattered SPP field from the NP. Within a 0th-order Born approximation, $E(\mathbf{r})$ may be written

$$
E(\mathbf{r})=E_{0}\left(\left|\mathbf{r}-\mathbf{r}_{1}\right|\right)+\alpha E_{0}\left(\left|\mathbf{r}_{2}-\mathbf{r}_{1}\right|\right) G\left(\left|\mathbf{r}-\mathbf{r}_{2}\right|\right),
$$

where $\mathbf{r}_{1}$ and $\mathbf{r}_{2}$ are the tip and NP locations, respectively. The cylindrical field propagating away from the tip is

$$
E_{0}\left(\left|\mathbf{r}-\mathbf{r}_{1}\right|\right)=e^{i k_{\mathrm{SPP}}\left|\mathbf{r}-\mathbf{r}_{1}\right|} / \sqrt{\left|\mathbf{r}-\mathbf{r}_{1}\right|,}
$$

where $k_{\mathrm{SPP}}=k^{\prime}+i k^{\prime \prime}$ is the complex SPP wave number. The propagator $G$ is proportional to the 0th-order Hankel function of the first type $H_{0}^{(1)}$ :

$$
G\left(\mathbf{r}, \mathbf{r}_{2}\right)=\frac{i}{4} H_{0}^{(1)}\left(k_{\mathrm{SPP}} d_{2}\right),
$$

where $d_{i}=\left|\mathbf{r}-\mathbf{r}_{\mathbf{i}}\right| . \quad H_{0}^{(1)}(x)$ tends asymptotically to $\sqrt{\frac{2}{\pi x}} e^{i\left(x-\frac{\pi}{4}\right)}$ for $x \gg 1$; [51] hence, far enough away from the NP (as compared to the SPP wavelength), the SPP field may be written:

$$
E(\mathbf{r})=e^{i k_{\mathrm{SPP}} d_{1}} / \sqrt{d_{1}}+A_{2} e^{i k_{\mathrm{SPP}} d_{2}} / \sqrt{d_{2}},
$$

where $A_{2}=\frac{\alpha}{4} \sqrt{\frac{2}{\pi k_{\mathrm{spd} d}}} e^{i\left(k_{\mathrm{spP}} d+\frac{\pi}{4}\right)}$ and $d=\left|\mathbf{r}_{2}-\mathbf{r}_{1}\right|$. Thus the SPP field intensity $I(\mathbf{r})=E(\mathbf{r}) E^{*}(\mathbf{r})$ may be written as a sum of three terms corresponding to the SPP fields emitted from the tip, from the NP and their interference, respectively, as follows:

$$
\begin{gathered}
I(r)=I_{\text {tip }}\left(d_{1}\right)+I_{\mathrm{NP}}\left(d_{2}\right)+I_{\text {int }}\left(d_{1}, d_{2}\right), \\
I_{\text {tip }}\left(d_{1}\right)=\frac{e^{-2 k^{\prime \prime} d_{1}}}{d_{1}}, \\
I_{\mathrm{NP}}\left(d_{2}\right)=\frac{|\alpha|^{2}}{8 \pi\left|k_{\mathrm{SPP}}\right| d d_{2}} e^{-2 k^{\prime \prime}\left(d+d_{2}\right)}, \\
I_{\text {int }}\left(d_{1}, d_{2}\right)=\frac{|\alpha|}{\sqrt{2 \pi\left|k_{\mathrm{SPP}}\right| d d_{1} d_{2}}} e^{-k^{\prime \prime}\left(d_{1}+d_{2}+d\right)} \\
\cos \left[k^{\prime}\left(d_{1}-d_{2}-d\right)-\frac{\pi}{4}-\arg (\alpha)+\frac{1}{2} \arg \left(k_{\mathrm{SPP}}\right)\right] .
\end{gathered}
$$

As an example, Fig. 2(d) in the main article shows the theoretical distribution of $I(\mathbf{r})$ for $d=3.9 \mu \mathrm{m}, \lambda_{0}=650 \mathrm{~nm}$, and $\arg (\alpha)=\frac{\pi}{2}$. Bright fringes exist in the interference pattern when the cosine in Eq. (A4) equals 1, i.e., whenever $d_{1}-d_{2}=d+\frac{1}{k^{\prime}}\left[2 \pi p+\frac{\pi}{4}+\arg (\alpha)-\frac{1}{2} \arg \left(k_{\mathrm{SPP}}\right)\right]$ where $p$ is an integer. As a result, the interference of the incident and scattered SPP waves yields a pattern of hyperbolic fringes. The angle $2 \Psi$ between the asymptotes of the bright fringes is given by $\cos (\Psi)=\frac{d_{1}-d_{2}}{d}$, i.e.,

$$
\cos (\Psi)=1+\frac{1}{k^{\prime} d}\left[2 \pi p+\frac{\pi}{4}+\arg (\alpha)-\frac{1}{2} \arg \left(k_{\mathrm{SPP}}\right)\right] .
$$

\section{APPENDIX B: MODEL FOR OUT-OF-PLANE (SPP-TO-PHOTON) SCATTERING}

From Eqs. (A2) and (A3), the intensity $I_{1}$ at the NP after SPP propagation from the tip and the intensity $I_{2}$ at the tip after SPP propagation back from the NP read:

$$
\begin{gathered}
I_{1}=\frac{I_{0}}{d} e^{-2 k^{\prime \prime} d} \\
I_{2}=\frac{|\alpha|^{2} I_{0}}{8 \pi\left|k_{\mathrm{SPP}}\right| d^{2}} e^{-4 k^{\prime \prime} d},
\end{gathered}
$$

where $I_{0}$ is the initial intensity of the electric field at the STM-tip nanosource.

The visibility of the fringe pattern in Fourier space reads:

$$
V=\frac{I_{\max }-I_{\min }}{I_{\max }+I_{\min }}
$$


The variation of $V$ with $d$ is the same in the two scenarios described in Figs. 3(c) and 3(d) (Sc. 1 and Sc. 2); indeed,

$$
V=2 \sqrt{\frac{\eta_{i}}{d}} \frac{e^{-k^{\prime \prime} d}}{1+\frac{\eta_{i}}{d} e^{-2 k^{\prime \prime} d}},
$$

where $\eta_{1}=\frac{\sigma_{1}}{\Gamma}$ in Sc. 1 and $\eta_{2}=\frac{|\alpha|^{2} \sigma_{2}}{8 \pi\left|k_{\mathrm{SPP}}\right| \sigma_{1}}$ in Sc. 2 .
Conversely, $I_{\max }$ varies quite differently with the tip-NP distance $d$, namely

$$
\begin{gathered}
I_{\max , \mathrm{Sc} .1}=\Gamma I_{0}\left(1+\sqrt{\frac{\eta_{1}}{d}} e^{-k^{\prime \prime} d}\right)^{2} \\
I_{\max , \mathrm{Sc} .2}=\sigma_{1} I_{0} \frac{e^{-2 k^{\prime \prime} d}}{d}\left(1+\sqrt{\frac{\eta_{2}}{d}} e^{-k^{\prime \prime} d}\right)^{2} .
\end{gathered}
$$

[1] E. Ozbay, Science 311, 189 (2006).

[2] H. A. Atwater, Sci. Am. 296, 56 (2007).

[3] K. F. MacDonald, Z. L. Samson, M. I. Stockman, and N. I. Zheludev, Nat. Photon. 3, 55 (2009).

[4] D. K. Gramotnev and S. I. Bozhevolnyi, Nat. Photon. 4, 83 (2010).

[5] V. J. Sorger, R. F. Oulton, R.-M. Ma, and X. Zhang, MRS Bull. 37, 728 (2012).

[6] E. Devaux, T. W. Ebbesen, J.-C. Weeber, and A. Dereux, Appl. Phys. Lett. 83, 4936 (2003).

[7] T. W. Ebbesen, C. Genet, and S. I. Bozhevolnyi, Phys. Today 61, 44 (2008).

[8] E. Devaux, J.-Y. Laluet, B. Stein, C. Genet, T. Ebbesen, J.-C. Weeber, and A. Dereux, Opt. Express 18, 20610 (2010).

[9] H. Ditlbacher, J. R. Krenn, G. Schider, A. Leitner, and F. R. Aussenegg, Appl. Phys. Lett. 81, 1762 (2002).

[10] J. R. Krenn, H. Ditlbacher, G. Schider, A. Hohenau, A. Leitner, and F. R. Aussenegg, J. Microsc. 209, 167 (2003).

[11] A. Drezet, A. Hohenau, A. L. Stepanov, H. Ditlbacher, B. Steinberger, F. R. Aussenegg, A. Leitner, and J. R. Krenn, Plasmonics 1, 141 (2006).

[12] A. B. Evlyukhin, S. I. Bozhevolnyi, A. L. Stepanov, R. Kiyan, C. Reinhardt, S. Passinger, and B. N. Chichkov, Opt. Express 15, 16667 (2007).

[13] I. P. Radko, A. B. Evlyukhin, A. Boltasseva, and S. I. Bozhevolnyi, Opt. Express 16, 3924 (2008).

[14] H. A. Atwater and A. Polman, Nat. Mater. 9, 205 (2010).

[15] C. M. Chang, M. L. Tseng, B. H. Cheng, C. H. Chu, Y. Z. Ho, H. W. Huang, Y.-C. Lan, D.-W. Huang, A. Q. Liu, and D. P. Tsai, Adv. Mater. 25, 1118 (2013).

[16] S. I. Bozhevolnyi, I. I. Smolyaninov, and A. V. Zayats, Phys. Rev. B 51, 17916 (1995).

[17] I. I. Smolyaninov, D. L. Mazzoni, and C. C. Davis, Phys. Rev. Lett. 77, 3877 (1996).

[18] B. Hecht, H. Bielefeldt, L. Novotny, Y. Inouye, and D. W. Pohl, Phys. Rev. Lett. 77, 1889 (1996).

[19] L. Novotny, B. Hecht, and D. W. Pohl, J. Appl. Phys. 81, 1798 (1997).

[20] J. Krenn, R. Wolf, A. Leitner, and F. Aussenegg, Opt. Commun. 137, 46 (1997).

[21] S. I. Bozhevolnyi and V. Coello, Phys. Rev. B 58, 10899 (1998).

[22] B. Vohnsen and S. I. Bozhevolnyi, Appl. Opt. 40, 6081 (2001).

[23] A. L. Stepanov, J. R. Krenn, H. Ditlbacher, A. Hohenau, A. Drezet, B. Steinberger, A. Leitner, and F. R. Aussenegg, Opt. Lett. 30, 1524 (2005).

[24] J. K. Day, O. Neumann, N. K. Grady, and N. J. Halas, ACS Nano 4, 7566 (2010).
[25] C. Lemke, T. Leiner, A. Evlyukhin, J. W. Radke, A. Klick, J. Fiutowski, J. Kjelstrup-Hansen, H.-G. Rubahn, B. N. Chichkov, C. Reinhardt, and M. Bauer, Nano Lett. 14, 2431 (2014).

[26] T. Coenen, F. Bernal Arango, A. Femius Koenderink, and A. Polman, Nat. Comm. 5 (2014).

[27] T. Coenen and A. Polman, ACS Nano 8, 7350 (2014).

[28] M. V. Bashevoy, F. Jonsson, A. V. Krasavin, N. I. Zheludev, Y. Chen, and M. I. Stockman, Nano Lett. 6, 1113 (2006).

[29] J. T. van Wijngaarden, E. Verhagen, A. Polman, C. E. Ross, H. J. Lezec, and H. A. Atwater, Appl. Phys. Lett. 88, 221111 (2006).

[30] N. Rotenberg, M. Spasenović, T. L. Krijger, B. le Feber, F. J. García de Abajo, and L. Kuipers, Phys. Rev. Lett. 108, 127402 (2012).

[31] N. Rotenberg, T. L. Krijger, B. le Feber, M. Spasenovic, F. J. de Abajo, and L. Kuipers, Phys. Rev. B 88, 241408(R) (2013).

[32] P. Bharadwaj, A. Bouhelier, and L. Novotny, Phys. Rev. Lett. 106, 226802 (2011).

[33] Y. Zhang, E. Boer-Duchemin, T. Wang, B. Rogez, G. Comtet, E. Le Moal, G. Dujardin, A. Hohenau, C. Gruber, and J. R. Krenn, Opt. Express 21, 13938 (2013).

[34] T. Wang, E. Boer-Duchemin, G. Comtet, E. Le Moal, G. Dujardin, A. Drezet, and S. Huant, Nanotechnology 25, 125202 (2014).

[35] T. Wang, G. Comtet, E. Le Moal, G. Dujardin, A. Drezet, S. Huant, and E. Boer-Duchemin, Opt. Lett. 39, 6679 (2014).

[36] Z. Dong, H.-S. Chu, D. Zhu, W. Du, Y. A. Akimov, W. P. Goh, T. Wang, K. E. J. Goh, C. Troadec, C. A. Nijhuis, and J. K. W. Yang, ACS Photon. 2, 385 (2015).

[37] S. Cao, E. Le Moal, E. Boer-Duchemin, G. Dujardin, A. Drezet, and S. Huant, Appl. Phys. Lett. 105, 111103 (2014).

[38] T. Wang, E. Boer-Duchemin, Y. Zhang, G. Comtet, and G. Dujardin, Nanotechnology 22, 175201 (2011).

[39] A. Bouhelier, T. Huser, H. Tamaru, H.-J. Güntherodt, D. W. Pohl, F. I. Baida, and D. Van Labeke, Phys. Rev. B 63, 155404 (2001).

[40] W. Abidi and H. Remita, Recent Pat. Eng. 4, 170 (2010).

[41] P. Johansson, R. Monreal, and P. Apell, Phys. Rev. B 42, 9210 (1990).

[42] A. Hohenau, J. R. Krenn, A. Drezet, O. Mollet, S. Huant, C. Genet, B. Stein, and T. W. Ebbesen, Opt. Express 19, 25749 (2011).

[43] A. B. Evlyukhin and S. I. Bozhevolnyi, Phys. Rev. B 71, 134304 (2005).

[44] A. Evlyukhin and S. Bozhevolnyi, J. Exp. Theor. Phys. Lett. 81, 218 (2005).

[45] R. Carminati, J. J. Sáenz, J.-J. Greffet, and M. Nieto-Vesperinas, Phys. Rev. A 62, 012712 (2000). 
[46] N. Dahan and J.-J. Greffet, Opt. Express 20, A530 (2012).

[47] C. Awada, G. Barbillon, F. Charra, L. Douillard, and J.-J. Greffet, Phys. Rev. B 85, 045438 (2012).

[48] A. Losquin, S. Camelio, D. Rossouw, M. Besbes, F. Pailloux, D. Babonneau, G. A. Botton, J.-J. Greffet, O. Stéphan, and M. Kociak, Phys. Rev. B 88, 115427 (2013).

[49] C. Symonds, A. Lemaître, E. Homeyer, J. C. Plenet, and J. Bellessa, Appl. Phys. Lett. 95, 151114 (2009).
[50] S. Aberra Guebrou, C. Symonds, E. Homeyer, J. C. Plenet, Y. N. Gartstein, V. M. Agranovich, and J. Bellessa, Phys. Rev. Lett. 108, 066401 (2012).

[51] M. Abramowitz and I. A. Stegun, eds., Hankbook of Mathematical Functions with Formulas, Graphs, and Mathematical Tables, Applied Mathematics Series, Vol. 55, 10th ed. (US Department of Commerce, National Bureau of Standards, Washington DC, 1972). 